parent that this congenital form of finger contraction is at in way allied to Dupuytren's finger contraction, from which it differs in its etiology and general pathology; in being a congenital affection, whilst Dupuytren's contraction belongs to adult life; in affecting chiefly the female instead of the male sex; and also in its anatomical characters, and difference in the structures involved, as neither the eentral portion of the palmar fascia, nor its lateral digitsl prolongations, are ever involved in the congenital form. The first phalanx is therefore never drawn down, bat as a rule is hyper-extended. With these differences are also correxponding differences in the principles of treatment. (To le concluded.)

\section{ON THE VALUE OF SUSPENSION IN THE TREATMENT OF TABES DORSALIS.}

BX \&. MICHELL CLARKE, M.A., M.B., M.R.C.P., ASTISTAMT IECTURER ON PHYSIOLOGY, BRISTOL MEDICAL SCHOOL, AND ASSISTANT PHYSICIAN AND PATHOLOGIST TO THE

$$
\text { GENERAL HOSPITAL. }
$$

IN this paper I propose to give the results of some obserrations nndertaken with the object of determining the value of suspension in the treatment of tabes dorsalis, and the mode in which it should be carried out in order to obtain the best results. The different observations cover the period extending from March 1st, 1889, to June 1st, 1891, so that sufficient time has elapsed in the majority of the cases treated to enable one not only to form a judgment as to the immediate effects of the treatment, whether beneficial or otherwise, but also to test whether these effects are lesting or merely temporary in character. The manner of carrying out suspension recommended by Prof. Charcot was followed. The patients were suspended regularly three times a wee? about an hour before their early dinner. On the first oxcasion the duration of suspension was thirty seconds only, and this was increased by thirty seconds at each saccessive isit until a period of three minutes was reached. In some of the earlier cases the duration was prolonged to Give minntes at each visit, and three patients were suspended by the head only instead of by the head and arms; but as a advantage was perceived to accrue either from the longer time of suspension or from the absence of the arm-slings, these modificstions were given up. In every case no medicine except antipyrin for pain, and occasional dyspeptic or aperient doses was given for from two to four months or longer after the treatment was begun. Later on, cod-liver oil and tonics were given to some of the patients, but in all a sufficient time was allowed to elapse to estimate the value of the treatment before other medicines were administered. With the exception of five patients, who were taken into the bospital on account of the distance at which their homes were situated, all were treated as out-patients, so that their ordinary condition and mode of life was not interfered with. Altogetber, twenty.nine cases were suspeaded for periods varying from one to ten months. Twentyfonx $\mathrm{w}$ ere cases of tabes dorsalis, two (brothers) of hereditary atary, two of spastic paraplegia, and one case of doubtful natare, probsibly of damage to cauda equina. ${ }^{1}$

It may be stated generally that no change was noted in any of the following symptoms in any case in which they were present: loss of knee-jerk or of plantar reflex ; Argyll.Robertson phenomenon, loss of dilatation of pupils to sensory stimu. lation, myosis, incouglity of pupils, oculo-motor palsy, or optic disc atrophy; paralysis of cranial nerves, atrophic paralysis of muscles (tongve and thenar eminence), or any considerable degree of anæsthesia. In one case the knee-jerks, which were feebly present at the commencement, became lost in the year subsequent to the treatment. The chief modes in which improvement was shown were in sense of general well-being, the patients nearly always stating that "they felt better in themselves" for suspension ; in increased strength and powers of walking; in lessened giddiness, but not always in the case of giddiness in the dark; in Romberg's symptom; in amelioration of lightning, rectal, and girdle pains ; in regaining partially or completely the sense of position of the limbs when that had been impaired or lost; and in improvement of feelings of numbness, tingling and formication, and light degrees of anasthesia. No con-

1 As acconnt of the more immediate effects of the treatment in inton of these cases will be found in the Practitioner, October, 1889. stant effect on disorders of micturition could be traced, some patients improving in this respect, the majority not. Where the occurrence of gastric crises was a marked feature of the illness, suspension did not diminish their frequency or severity, and in some cases seemed to make them worse at the commencement. Of accidents there were none. The treat. ment was interrupted or stopped in a few cases on account of attacks of sickness; in one patient odema of the feet supervened without any definite cause ; in several giddiness for a few minutes after suspension was troublesome ; but only one patient was sufficiently bad to make it advisable to stop treatment altogether. One man (No. 18), who had suffered two years previously from paralysis of the abductors of the vocal cords, for which tracheotomy had been recommended but not performed, and who still had marked paresis of these muscles, bore suspension without any discomfort. Of the twenty-four cases of tabes dorsalis two were men suffering from advanced atrophy of optic discs-one of them was blind and in the other vision was greatly impaired-together with absent knee-jerks and lightning pains, but no other cord symptoms. These patients were suspended on account of the published statement that the treatment had resulted in improved vision in such cases. Unfortunately no such effect was produced in my two patients. Of the other twenty-two cases the result was good in three cases, and in them the benefit has so far remained permanent. Two others also derived great good from prolonged courses of suspension, but relapsed after an interval of six months, becoming nearly as bad as before; both these patients returned, and were subjected to a second course of two months' duration from which they gained as much benefit as before, and have now remained well for about six months. Five patients were improved, but to a less degree than the foregoing, Of these two have been lost sight of ; one relapsed after a time, and was getting steadily worse when last heard of one relapsed after eight months of good health and hard work, and is again improving rapidly under a second course, and another went back to some degree, though he did not become so bad as he was before suspension, and recently returned for fresh treatment. In this last case the duration of the first course of suspension was only a month, and he has again derived good from a second course. Two other cases must be put down as slightly improved but are still under my care, and therefore the final result cannot be stated. In two more slight improvement has been shown as regards some symptoms, and they are so far better and have remained so for some time-a year and a half in one case, - but in most respects their condition is unchanged. Another patient improved at the commencement of treatment only, and must be classed with the seven remaining cases in which the result was absolutely nil.

I have thought it desirable in the further analysis of the cases to divide them according as they fell into one of the three groups or stages now generally recognised as belonging to tabes dorsalis. These are-(1) the pre-ataxic stage in which ataxy of movement is not present or is only very slightly marked, but the existence of the disease is shown by the occurrence of more or fewer of the following sym. ptoms : loss of knee-jerk or of other tendon reflexes, inequality of pupils, Argyll - Robertson phenomenon, myosis, atrophy of optic discs, oculo-motor paralyses, Romberg's sy mptom, lightning pains, gastric crises, disorders of micturition, affections of common sensation, girdle pain, \&c.; (2) the ataxic stage, in which locomotor ataxy is a striking feature; and (3) the stage in which the patient is either unable to walk at all or only with great difficulty with the aid of others. I may state parenthetically that it is not meant to imply by the expression "stages" that the first must necessarily pass into the second stage, for many cases remain in the first stage and never develop marked ataxy. Of the twenty-four patients, twelve were in the pre-ataxic stage, and the following table gives the result with the duration of the disease in years :-

Duration of No. of Result.

1 year ... 1... Good, but relapsed, improving again under

In one good, another improved, in two nil. Good.

(One improved, one better at first, but relapsed at once, and in the third improvement, but still under treatment.

$10, \ldots \quad \ldots .1$. $1 .$. Improsed. Nil.

Good; relapsed and improved again.

Total result: Good in four, two, however, relapsing and 
Table of 29 Cases of Suspension in the Treatment of Tabes Dorsalis.

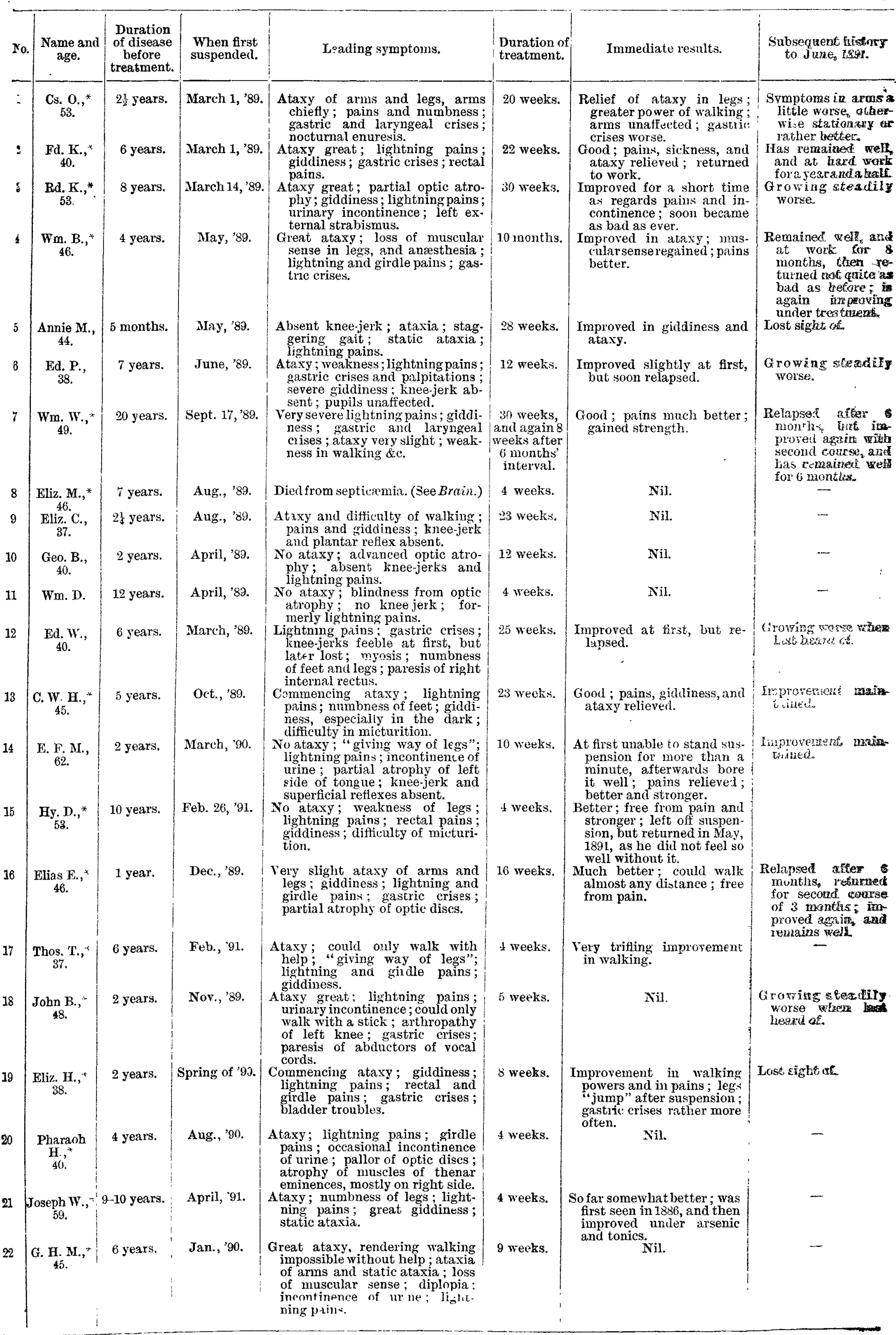


Treatment of Tabes Dorsalis.-(Continued)

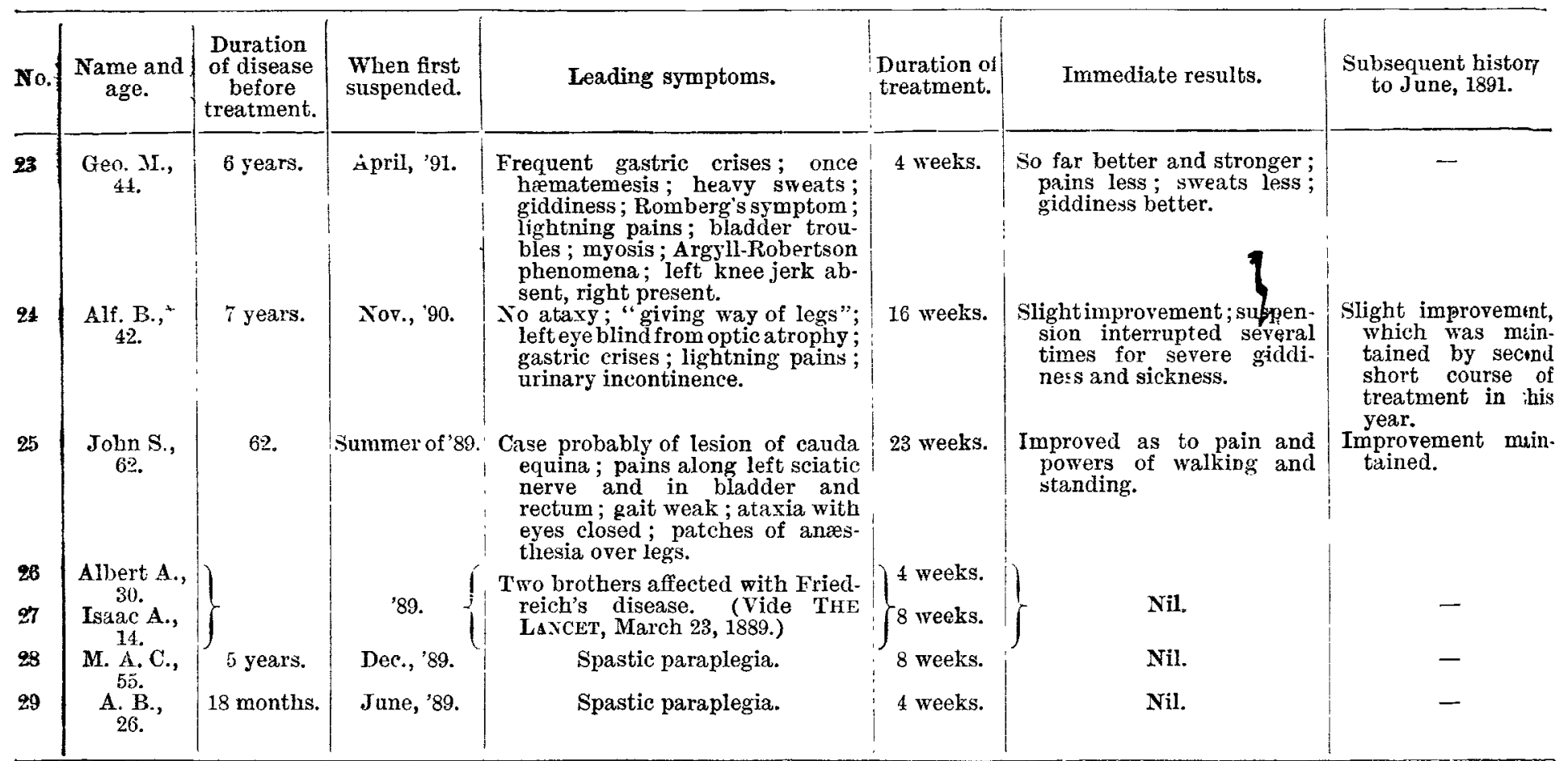

Note.-In the table, an asterisk after the patient's name indicates, for sake of brevity, that the knee-jerk was absent, the Argyll-Roberson phenomena present, and that there was either myosis or inequality of pupils. Case 1 is reported fully in Brain, spring, number, 1891 ; Case 8 in Brain, autumn number, 1890 ; Cases 26 and 27 in 'THE LANCET, March 23 rd, 1889, and a fuller account of tirst 12 cases in Practitioner, Oct., 1889.

improving under second course; improvement in three; nil in four; one too soon to judge of the lasting result. Eleven were in the ataxic stage, and are arranged according to the duration of the disease.

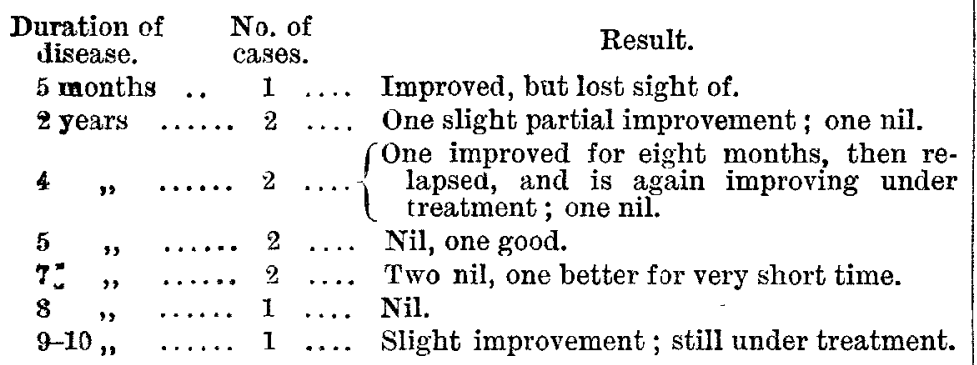

$9-10, \ldots . .1 \quad 1 .$. Slight improvement; still under treatment.

Total result: Good, one; improved, three, of whom one relapsed; sligtly improved, one ; nil, six.

In the first case marked as good, a very satisfactory result was attained after sixty suspensions; he suffered from severe ataxy, lightning pains, and occasional incontinence of urine, quite incapacitating him from work. After the treatment he was able to go back to his work at a drilling machine, and has remained well and at work for nearly a year and a half, suffering occasionally only from slight pains and giddiness. One patient in the third stage gained no advantage from a course of suspension lasting nine weeks. As to the length of time during which suspension was carried out: this was ten months in one case, twenty-five to thirty weeks in four cases, twenty to twenty-three weeks in another four, sixteen weeks in three, eight to twelve weeks in five, and four to five weeks in seven cases. The periods may seem very long, but the treatment was purposely extended in order to ascertain whether the effects would increase in direct ratio with the length of time. On carefully comparing the results attained with the duration of treatment, I have come to the conclusion that no proportionate advantage was gained from the very long courses of suspension, and that from ten to twelve weeks-or at most sixteen weeks-is long enough in all cases. After twelve weeks all the benefit that is to be gained from it will have been gained, and if there is no improvement after eight weeks' treatment there is not likely to be any afterwards. If a relapse occurs after some months a second course of eight to twelve weeks' duration may be carried out with good results, as is shown in some of the above cases. Some importance is to be attached to the degree of care with which suspension is performed. I believe some of the failures are due to carelessness in the manner of doing it. The straps should be carefully adjusted, so that the head and arms may each bear their due share of the weight of the body; the supports should be properly padded, in order to avoid giving discomfort to the patient, and the head-supports be co arranged that they do not compress the vessels of the neck. I feel sure that attention to minor points will often make all the difference in the results gained from the treatment. As to the other patients, the two cases of spastic paraplegia and of Friedreich's disease remained unaffected for good or bad, so far as could be ascertained. The man suffering from damage to cauda equina improved very much both as to gait, pains, and numbness over the legs, and has remained much better ever since.

The manner in which suspension acts is a difficult problem to solve. The structures which support and protect the spinal cord would seem especially adapted to prevent any actual stretching of it whilst they remain intact, and it appears improbable that suspension should cause changes in the circulation of the blood through the cerebro-spinal axis sufficiently profound to produce lasting effects. It is suggestive that the best results were arrived at in those patients in whom the disease had not gone on to the development of ataxy, and that a larger proportion of them improved than of the ataxic. In this class lightning pains are often most intense and recur at frequent intervals, rendering the patient's life a miserable one. Among the other clinical features are weakness of the legs and an inability to walk any distance, whilst general muscular feebleness is often as marked a symptom in them as not infrequently it is conspicuously absent in cases that show great ataxy of movement. Together with the other signs that place the existence of tabes dorsalis beyond doubt, there is often a general feebleness, loss of energy, and want of confidence in their powers. These patients are afraid to cross a busy street or to go in to crowded places. One cannot in the consideration of tabes dorsalis leave out of sight the disturbing influence of the morbid changes in the cord on cerebral and cerebellar processes, and, again, the secondary effects of this disturbance on the mind and emotions. In short, the greater part of the central nervous system is directly or indirectly concerned, and we may suppose that according to the individual susceptibility to such secondary effects their extent and intensity will vary in particular cases. Or in other words, in some cases the remote functional derangements will be out of proportion to the actual organic changes. Though the structural lesion interferes directly with the proper performance of certain functions, indirectly it has a still wider influence. The partial or complete absence of certain sensations that normally guide us in the functions of station, locomotion, and other coördinated muscular actions induces the patient to believe that he is incapable of performing much that is in reality 
still possible for him. He ceases to be able to distinguish what he can from what he cannot do, loses confidence in his powers, becomes nervous as regards all motor actions, probably often fails from concentrating his attention too strongly upon the execution of them, so that the range of disablement is indefinitely extended. Could we, however, restore his confidence, make him feel that he was regaining bis former powers of easy coördination of muscular action, we should relieve him of many of his symptoms-namely, those indirectly or remotely connected with the actual lesion, although the direct signs of structural mischief in the cord would remain unchanged. Now, there can be no doubt that the patients do thoroughly believe in suspension, and in some unexplained way they regain during treatment their lost confidence in their own powers. When they improve their first statement is that they feel better and stronger, have a sense of improved well-being, can do more, walk further, step ont more vigorously, and no longer fear to go into crowded streets. Later on, as the next most constant signs of benefit, we have relief of pains, of giddiness, and perhaps of ataxy. On the other hand, the physical signs of organic disease remain unaffected. It is thus by busying itself on the more indirect symptoms that suspension appears to me to act, but in what manner I am quite unable to suggest. I believe that in the feeling of general well-being and of regained power of coördination of movement that it induces its real modus operand $i$ is to be sought.

In a disease like tabes dorsalis, where the progress of the disorder is attended by frequent remissions or may be arrested at almost any stage, it is difficult to estimate aright the value of any particular mode of treatment, but I think that the following conclusions are justified: -1 . That suspension is capable of affording relief in a certain proportion of cases of tabes dorsalis, and that cases in the "preataxic" stage derive the most benefit from it. Where locomotor ataxy has progressed so far as to prevent walking it is useless. 2. The duration of the disease appears to have little influence on the result, but the prospect of relief is somewhat more favourable in those in whom it has not lasted over six years. 3. If it is carefully carried out no untoward results need be feared ; but in patients who suffer severely from gastric crises its use demands caution. 4. A twelve-weeks' course of suspension is likely to give as good results as one of more prolonged duration, but it should be continued for this length of time. 5 . A relapse may occur after the patient has remained well for some time, and then fresh benefit may be derived from a second course of from eight to twelve weeks.

Clifton, Bristol.

\section{CASE OF "SKEWER" AMPUTATION OF THE ENTIRE UPPER EXTREMITY.}

By BRIG. SURG. K. MCLEOD, M.D., F.R.C.S. EdiN., PROFESSOR OF SURGERY, CALCUTTA MEDICAI COLLEGE.

Is ThE LANCET of April 19th, 1890 (page 847), I described the use of a combination of skewers and elastic cord for the purpose of preventing hæmorrhage in removing large tumours. I mentioned in that paper a case of amputation of the entire upper extremity by Mr. Joseph Bell of Edinburgh, in which the use of this combination had contributed to a brilliant success, so far as recovery from the operation was concerned. I have recently performed a similar operation, the success of which I attribute to the employment of similar measures. The details of the case are as follows:-

$\mathrm{H}$ - , a Mahomedan female aged fifteen, was admitted into the Medical College Hospital on the 21st of March, 1891. About three months before admission she noticed a swelling of the right arm and shoulder, which has continued to increase rapidly. Beyond the pain and in. convenience caused by the growth, her general health and comfort have not been injuriously affected. On admission a large globular tumour was observed beneath the jight deltoid muscle, which was tightly stretched over it. Its lower limit corresponded to the insertion of the deltoid, and it reached the top of the shoulder, the acromion process being imperceptible. The circumference of the limb at the shoulder was nineteen inches, and the vertical arc of the tumour measured eight inches and a half. The growth was firm at its circumference, but soft and fluctuating at its centre. It filled the axillary space. The scapula was evidently implicated, and no movement existed at the shoulder-joint, the scapula and clavicle moving with the tumour. The outer end of the clavicle was lost in the mass. The skin over the tumour was tightly stretched and cedematous, but not infiltrated or broken. The tumour was tender to pressure and manipulation. The right arm and forearm were cedematous, and the right pulse somewhat weaker than the left. Patient was fairly nourished and had a healthy colour. Lungs and heart healthy; liver and spleen of normal size. Slight pyrexia $\left(100^{\circ} \mathrm{F}\right.$.) occurred towards evening, but beyond sleeplessness and slight indigestion she was in the enjoyment of good general health.

An operation was performed on March $23 \mathrm{rd}$ for the removal of the mass, which was evidently a sarcoma, originating in the head of the humerus, and secondarily implicating the adjoining portion of the scapula and the muscles inserted into the head of the humerus. The part having been thoroughly cleansed and the patient chloroformed, a puncture was made in the floor of the axilla and another above and behind the tip of the shoulder, and a stout packing needle, about eight inches long, was passed from below upwards through the cavity of the axilla, behind the vessels and nerves, within the girdle of the shoulder bones, and finally made to emerge through the second puncture. A second needle of the same size was entered at the lower puncture, directed transversely beneath the scapula, and made to emerge behind the posterior or vertebral border of the bone. A strong indiarubber cord was wound, figure of 8 fashion, round the projecting ends of these needles, encircling the part with a tight elastic loop which was held in position by these skewers. A circular incision was now made about two inches on the distal aspect of this loop. It was first deepened in front, and the clavicle was reached and divided at the junction of the middle and outer thirds. The plexus and vessels were thus exposed and they were isolated, and a clamp forceps put on them for additional security. The attachments of the scapula were then rapidly divided and the extremity removed. The arrangements for preventing hæmorrhage were perfectly effective. The axillary artery was now identified, isolated, and tied; the elastic cord gradually loosened, and the vessels as they presented were secured. The skewers were finally withdrawn and the nerves cut short. The axillary glands were removod en masse. Two drainage-tubes were inserted, one at the lower angle of the wound, and the other through an opening made where the lower angle of the scapula had rested. The flaps were stitched with silk and horsehair. They were rather scrimp, and required a little traction to bring them together. The wound was dressed with scrupulous antiseptic precautious, and the patient, who exhibited some signs of shock, ordered frequent doses of a diffasible stimulant. During the first few days the pulse remained abnormally rapid and the stomach very irritable. Patient was sleepless and complained of severe pain in the epigastrium. The morning temperature was normal, and there was a slight rise to $99^{\circ}$ or $100^{\circ}$ towards evening for a week. The wound remained aseptic. Some suppuration occurred in the track of the posterior tube. The tubes were gradually shortened and finally withdrawn on the lst and 3rd of April. The lips of the wound united, but there was some tension of the deep stitches, which began to cut, when they were withdrawn. The granulation material stretched to the extent of about an inch and a half. The wound eventually healed by granulation, the cicatrix being linear and freely movable over the ribs.

The tumour was found by Dr. Gibbons to be a spindlecelled sarcoma, growing and degenerating very rapidly. Having previously performed this operation, with the result of death from hrmorrhage and shock on the same day, the favourable issue in the present instance was peculiarly gratifying to me. The subject was certainly more promising than either of the other two, but the signs of shock were very evident and somewhat alarming on this oceasion, and had loss of blood been superadded, it is almost certain that death would have taken place. The skewers and cord were applied with very great ease and speed, and the control which they exercised over the bleeding was absolute. The arrangement also permitted of gradual relaxation of the cord, and the vessels requiring deligation were thus secured at:ilt:in. Calcutta. 